\title{
Stability of Stochastic Logistic Model with Ornstein-Uhlenbeck Process for Cell Growth of Microorganism in Fermentation Process
}

\author{
Tawfiqullah Ayoubi \\ School of Mathematics and Statistics, Southwest University, Chongqing, China \\ Email: tawfiqullah_ayuobi@yahoo.com
}

How to cite this paper: Ayoubi, T. (2019) Stability of Stochastic Logistic Model with Ornstein-Uhlenbeck Process for Cell Growth of Microorganism in Fermentation Process. Applied Mathematics, 10, 659-675. https://doi.org/10.4236/am.2019.108047

Received: July 2, 2019

Accepted: August 10, 2019

Published: August 13, 2019

Copyright ( 2019 by author(s) and Scientific Research Publishing Inc. This work is licensed under the Creative Commons Attribution International License (CC BY 4.0).

http://creativecommons.org/licenses/by/4.0/

\section{(c) (i) Open Access}

\begin{abstract}
Current research is concerned with the stability of stochastic logistic equation with Ornstein-Uhlenbeck process. First, this research proves that the stochastic logistic model with Ornstein-Uhlenbeck process has a positive solution. After that, it also introduces the sufficient conditions for stochastically stability of stochastic logistic model for cell growth of microorganism in fermentation process for positive equilibrium point by using Lyapunov function. In addition, this research establishes the sufficient conditions for zero solution as mentioned in Appendix $\mathbf{A}$ due to the cell growth of microorganism $\mu_{\max }$, which cannot be negative in fermentation process. Furthermore, for numerical simulation, current research uses the 4-stage stochastic Runge-Kutta (SRK4) method to show the reality of the results.
\end{abstract}

\section{Keywords}

Stability, Fermentation Process, Ornstein-Uhlenbeck Process, Logistic Model, Lyapunov Function, 4-Stage Stochastic Runge-Kutta Method

\section{Introduction}

Stochastic differential equations (SDEs) have been intensively used to model the natural phenomena in the last decades and these equations play a prominent applied role in various fields [1] [2]. Most SDEs do not have explicit solution but these equations can be solved numerically to approximate their solutions [3]. We use the SRK method to approximate the solution numerically ([3], p. 69) [4] [5] [6] [7]. Whereas, Rüemelin in [8] proposed the $S$-stage stochastic Runge-Kutta explicit method. Moreover, Xiao in [9] introduced the High strong order stochastic Runge-Kutta methods for stochastic differential equations in case of 
Stratonovich since our model is in Stratonovich sense.

This research deals with the stability of stochastic logistic model with Ornstein-Uhlenbeck process. This process is a batter model of Brownian motion ([10], p. 86). The Ornstein-Uhlenbeck process is used to model stock price, currency exchange rates and velocity.

The classical Brownian motion was introduced by Scottish Botanist Robert Brown in (1827). He described this motion based on random movements of pollen grains in liquid or gas [11]. This theory was further supported by Norbert Wiener (1923) who explained full mathematical theory of Brownian motion which existed as a rigorously defined mathematical objective to recognize his contribution [12]. Brownian motion is a simple continuous stochastic process which is extensively used to model phenomena in various fields such as industry, dynamic process, physics, finance and fermentation process [3] [11] [12]. This Brownian motion is the cause of instability (turbulence) in fermentation process [1].

Fermentation process converts sugar into alcohol with the help of yeast [13]. Madihah, in [14] used the logistic model to elaborate the cell growth of Clostridium acetobutylicum p. 262 in fermentation process proposed by Verhulst (1838). This model is inadequate to describe the cell growth of microorganism in fermentation process because fermentation process contains random fluctuations. Bahar and Mao in [2] introduced new stochastic logistic model for population dynamics. The stochastic logistic model was used in [15] to elucidate the cell growth of Clostridium acetobutylicum p. 262 in fermentation process. This cell growth of microorganism in fermentation process is affected by environmental noise, a batter model for modelling environmental noise is Ornstein-Uhlenbeck process. However, no specific research was conducted on stability stochastic logistic model with Ornstein-Uhlenbeck process for cell growth of microorganism in fermentation process. Nevertheless, there are some previous research works which have been done on stability of logistic equations with white noise, Lévy Jumps and other different aspects [16]-[22]. None of these researches were investigated the stability of stochastic logistic model with Ornstein-Uhlenbeck process for cell growth of microorganism in fermentation process. This research establishes the sufficient conditions for stochastic logistic equation with Ornstein-Uhlenbeck process for positive equilibrium point and zero solution by using Lyapunov function. Since, the Ornstein-Uhlenbeck process is a better model of Brwonian motion ([10], p. 86). In addition, our current study will indicate that the Ornstein-Uhlenbeck process is unfavorable for stability of cell growth in fermentation process. Moreover, we apply the SRK4 method to evaluate the numerical solution.

This paper is organized in five main sections; Introduction, Preliminaries and Models Description, Main Results, Numerical Simulation and Conclusion.

\section{Preliminaries and Models Description}

Throughout this paper; the notation $E[x(t)]$ is the expectation of $(x(t)), T$ shows the terminal time, $t$ is time, $t_{0}$ illustrates the initial time, $x(t)$ corresponds to the highest cell size, $x_{0}$ is initial cell size, $\mu_{\max }$ denotes the maxi- 
mum specific growth rate, $x_{\max }$ illustrates a carrying capacity, $\sigma$ indicates the random fluctuation, $W(t)$ shows Brownian motion and $b$ is a constant.

The simplest mathematical model to illustrate the exponential phase for cell growth in fermentation process is:

$$
\frac{\mathrm{d} x(t)}{\mathrm{d} t}=\mu_{\max } \times x(t) .
$$

The solution of Equation (1) is:

$$
x(t)=x_{0} \mathrm{e}^{t \times \mu_{\max }},
$$

where $t$ is time, $x_{0}$ corresponds to the initial cell size and $\mu_{\max }$ denotes the maximum specific growth rate. If $\mu_{\max }>0$, Equation (1) is strongly ascending, and if, $\mu_{\max }<0$, strongly descending. Hence, Equation (1) is not adequate to model stationary phase in fermentation. Therefore, Pierre Francois Verhulst (1838) introduced new model containing stationary phase in fermentation process. Thus, the exponential growth model (1) is augmented by the inclusion of multiplicative factor of $1-\frac{x(t)}{x_{\max }}$. Hence, the logistic ordinary differential equation is:

$$
\mathrm{d} x(t)=\mu_{\max }\left(1-\frac{x(t)}{x_{\max }}\right) x(t) \mathrm{d} t
$$

where $x_{\max }$ is a carrying capacity of a microbial species and Equation (3) can be solved analytically by determining the solution is:

$$
x(t)=\frac{x_{\max } x_{0} \mathrm{e}^{\mu_{\max } t}}{x_{\max }+x_{0}\left(-1+\mathrm{e}^{\mu_{\max } t}\right)},
$$

where $x(t)$ is the highest cell size. Madihah, in [14] used Equation (3) to elaborate cell growth of Clostridium acetobutylicum p. 262 in batch fermentation process. Murray in [23] and May in [24] were proved that Equation (3) is stable. This model is inadequate to describe the cell growth of Clostridium acetobutylicum p. 262 in fermentation process because fermentation process suffers from random fluctuation. Bahar and Mao in [2] introduced new stochastic logistic equation to model uncontrolled fluctuation in stationary phase at fermentation process by using new perturbation very randomly which is:

$$
\rho \rightarrow \rho+\sigma \frac{\mathrm{d} W(t)}{\mathrm{d} t}
$$

where $\rho=\frac{\mu_{\max }}{x_{\max }}$ is diffusion coefficient. Model (3) with perturbation (5) is:

$$
\mathrm{d} x(t)=\mu_{\max }\left(1-\frac{x(t)}{x_{\max }}\right) x(t) \mathrm{d} t+\sigma x^{2}(t) \mathrm{d} W(t), t \in[0, T],
$$

where $W(t)$ is $m$-dimensional Weiner process and $\sigma$ is corresponding to the random fluctuation. Bazli, in [15] used Equation (6) to evaluate the cell growth of Clostridium acetobutylicum p. 262 in fermentation process.

Remarks 2.1: Liu et al., in [21] studied the stability of stochastic logistic with 
white noise which cannot model random fluctuations in stationary phase. Therefore, this research introduces new stochastic logistic model to cope with this problem and established the sufficient condition for positive equilibrium point. The new model is Equation (6) with Ornstein-Uhlenbeck process. The Ornstein-Uhlenbeck process is:

$$
y^{\prime \prime}(t)=-b y^{\prime}(t)+\mathrm{d} W(t)
$$

where $y(t)$ stands for positive Brownian motion at time $t, b>0$ shows the coefficient friction, $\sigma$ indicates the diffusion coefficient and $W(t)$ is white noise.

By substituting

$$
y^{\prime}(t)=W(t),
$$

into Equation (7) hence, the Ornstein-Uhlenbeck process becomes;

$$
\mathrm{d} W(t)=-b W(t) \mathrm{d} t+\sigma \mathrm{d} W(t) .
$$

Substituting Equation (9) into Equation (6) yields

$$
\mathrm{d} x(t)=\mu_{\max }\left(1-\frac{x(t)}{x_{\max }}\right) x(t) \mathrm{d} t-\sigma x^{2}(t) b W(t) \mathrm{d} t+x^{2}(t) \sigma^{2} \mathrm{~d} W(t) .
$$

Equation (10) is stochastic logistic model with Ornstein-Uhlenbeck process.

\section{Main Results}

First, it is needed to prove that Equation (10) has a unique positive solution and later on we will pay attention on stability.

Theorem 3.1: For all $t \geq 0$ and for any $0<x(0)=x_{0}$ Equation (10) has a unique positive solution.

Proof: For any given initial value $x_{0} \in R_{+}^{n}$, the coefficients of Equation (10) are locally Lipschitz continuous. Hence, there is a unique locally solution $x(t), t \in\left[0, i_{e}\right)$ Where $i_{e}$ shows the explosion time Arnold in [25] and Friedman [26]. To show that the $x(t), t \in\left[0, i_{e}\right)$ is a unique positive solution. So, it is really necessary to determine that $i_{e} \rightarrow \infty$. Let $k_{0} \in\left[\frac{1}{k_{0}}, k_{0}\right], k_{0}>0$ and $k>k_{0}$ is sufficiently large for each component of $x_{0}$, the stopping time for every integer $k>k_{0}$ is:

$$
t_{k}=\inf \left\{t \in\left[0, i_{e}\right): x(t) \text { is not in }\left(\frac{1}{k}, k\right)\right\} .
$$

$k_{0}$ is sufficiently large means that the $t_{k}$ is increasing as $k \rightarrow \infty$. Hence, $t_{\infty}=\lim _{k \rightarrow \infty} t_{k}$, whereas $t_{\infty} \leq i_{e}$.

If we can show that $i_{\infty}=\infty$ a.s., then $i_{\infty}=\infty$ a.s. and $x(t) \in R_{+}^{n}$ a.s. for all $t \geq 0$. In other words, to complete the proof all we need to show is that $i_{\infty}=\infty$ a.s. For if this statement is false, then there is a pair of constants $T>0$ and $\epsilon \in(0,1)$ such that

$$
P\left\{i_{\infty} \leq T\right\} \geq \epsilon
$$


Therefore, there exist an integer $k_{1} \geq k_{0}$

$$
P\left\{i_{\infty} \leq T\right\} \geq \epsilon, \forall k \geq k_{1}
$$

Define $V(x(t))=\left(\sqrt{x(t)}-1-\frac{1}{2} \ln x(t)\right)$ for all $x(t) \in\left[0, i_{e}\right)$ and the nonnegativity of this function can be seen from

$$
\sqrt{u}-1-\frac{1}{2} \ln u
$$

Itô ([27], p. 95) [28] [29] [30] ([31], p. 54) formula shows that

$$
\begin{aligned}
\mathrm{d}(V(x(t)))= & \frac{1}{2 \sqrt{x(t)}}-\frac{1}{2 x(t)}\left[\mu_{\max }\left(1-\frac{x(t)}{x_{\max }}\right) x(t) \mathrm{d} t\right] \\
& -\underbrace{\left[\sigma x^{2}(t) b W(t) \mathrm{d} t+\sigma^{2} x^{2}(t) \mathrm{d} W(t)\right]}_{A} \\
& +\underbrace{0.5\left[\frac{0.25}{\sqrt{x^{3}(t)}}-\frac{1}{2 x^{2}(t)}\left(\mu_{\max }\left(1-\frac{x(t)}{x_{\max }}\right) x(t) \mathrm{d} t\right)\right.}_{B} \\
& -\underbrace{\left.\sigma x^{2}(t) b W(t) \mathrm{d} t+\sigma^{2} x^{2}(t) \mathrm{d} W(t)\right]^{2}}_{B}
\end{aligned}
$$

To simplify $A$ and $B$ separately yields

$$
\begin{aligned}
& A=\frac{1}{2 \sqrt{x(t)}} \mu_{\max }\left(1-\frac{x(t)}{x_{\max }}\right) x(t) \mathrm{d} t-\frac{1}{2 \sqrt{x(t)}} \sigma x^{2}(t) b W(t) \mathrm{d} t \\
& +\frac{1}{2 \sqrt{x(t)}} \sigma^{2} x^{2}(t) \mathrm{d} W(t)-\frac{1}{2 x(t)} \mu_{\max }\left(1-\frac{x(t)}{x_{\max }}\right) x(t) \mathrm{d} t \\
& +\frac{1}{2 x(t)} \sigma x^{2}(t) b W(t) \mathrm{d} t-\frac{1}{2 x(t)} \sigma^{2} x^{2}(t) \mathrm{d} W(t) \\
& B=\frac{0.25}{\sqrt{x^{3}(t)}}-\frac{1}{2 x^{2}(t)}\left[\left(\mu_{\max }\left(1-\frac{x(t)}{x_{\max }}\right) x(t) \mathrm{d} t\right)^{2}\right. \\
& +2\left(\mu_{\max }\left(1-\frac{x(t)}{x_{\max }}\right) x(t) \mathrm{d} t\right)\left(-\sigma x^{2}(t) b W(t) \mathrm{d} t+\sigma^{2} x^{2}(t) \mathrm{d} W(t)\right) \\
& \left.+\left(-\sigma x^{2}(t) b W(t) \mathrm{d} t+\sigma^{2} x^{2}(t) \mathrm{d} W(t)\right)^{2}\right] \\
& B=\frac{0.25}{\sqrt{x^{3}(t)}}-\frac{1}{2 x^{2}(t)}\left[\left(\mu_{\max }^{2} x^{2}(t) \mathrm{d} t \times \mathrm{d} t-2 \frac{\mu_{\max }^{2}}{x_{\max }} x^{2}(t) \mathrm{d} t \times \mathrm{d} t\right.\right. \\
& \left.+\left(\frac{\mu_{\text {max }}}{x_{\max }} x(t) \mathrm{d} t\right)^{2}\right)+2\left(\mu_{\text {max }} x(t) \mathrm{d} t-\frac{\mu_{\text {max }}}{x_{\text {max }}} x^{2}(t) \mathrm{d} t\right) \\
& \times\left(-\sigma x^{2}(t) b W(t) \mathrm{d} t+\sigma^{2} x^{2}(t) \mathrm{d} W(t)\right)+\left(-\sigma x^{2}(t) b W(t) \mathrm{d} t\right)^{2} \\
& \left.+2\left(-\sigma x^{2}(t) b W(t) \mathrm{d} t\right)\left(\sigma^{2} x^{2}(t) \mathrm{d} W(t)\right)+\left(\sigma^{2} x^{2}(t) \mathrm{d} W(t)\right)^{2}\right]
\end{aligned}
$$


An application of these facts $\mathrm{d} t \times \mathrm{d} t=0, \mathrm{~d} t \times \mathrm{d} w(t)=0$ and $\mathrm{d} W(t) \times \mathrm{d} W(t)=\mathrm{d} t$ ([32], p. 87) and somewhat lengthy calculation we have

$$
\begin{gathered}
B=\frac{0.125}{\sqrt{x^{3}(t)}-\frac{0.25}{x^{2}(t)}\left(\sigma^{4} x^{4}(t) \mathrm{d} t\right)} \\
\Rightarrow B=\frac{0.125 \sigma^{4} x^{4}(t) \mathrm{d} t}{\sqrt{x^{3}(t)}}-\frac{0.25 \sigma^{4} x^{4}(t) \mathrm{d} t}{x^{2}(t)}
\end{gathered}
$$

By substituting the value of $A$ and $B$ into Equation (12) yields

$$
\begin{aligned}
& \mathrm{d}(V(x(t)))=\left[\frac{1}{2 \sqrt{x(t)}} \mu_{\max }\left(1-\frac{x(t)}{x_{\max }}\right) x(t) \mathrm{d} t-\frac{1}{2 \sqrt{x(t)}} \sigma x^{2}(t) b W(t) \mathrm{d} t\right. \\
& +\frac{1}{2 \sqrt{x(t)}} \sigma^{2} x^{2}(t) \mathrm{d} W(t)-\frac{1}{2 x(t)} \mu_{\max }\left(1-\frac{x(t)}{x_{\max }}\right) \mathrm{d} t \\
& \left.+0.5 \sigma x(t) b W(t) \mathrm{d} t-0.5 \sigma^{2} x(t) \mathrm{d} W(t)\right] \\
& +\frac{0.125 \sigma^{4} x^{4}(t) \mathrm{d} t}{\sqrt{x^{3}(t)}}-\frac{0.25 \sigma^{4} x^{4}(t) \mathrm{d} t}{x^{2}(t)} \\
& \mathrm{d}(V(x(t)))=\left[0.5 x^{0.5}(t) \mu_{\max }\left(1-\frac{x(t)}{x_{\max }}\right)-0.5 x^{-0.5}(t) \mu_{\max }\left(1-\frac{x(t)}{x_{\max }}\right)\right. \\
& -0.5 \sigma x(t) b W(t)+0.5 \sigma x(t) b W(t)+0.125 \sigma^{4} x^{2.5}(t) \\
& \left.-0.125 \sigma^{4} x^{2}(t)\right] \mathrm{d} t-0.5 \sigma^{2} x^{2}(t) \mathrm{d} W(t) \mathrm{d} t-0.5 \sigma^{2} x(t) \mathrm{d} W(t) \\
& \mathrm{d}(V(x(t)))=\left[0.5 x^{0.5}(t) \mu_{\max }\left(1-\frac{x(t)}{x_{\max }}\right)-0.5 x^{-0.5}(t) \mu_{\max }\left(1-\frac{x(t)}{x_{\max }}\right)\right. \\
& \left.+0.5 \sigma x(t) b W(t)\left(1-x^{0.5}(t)(t)\right)+0.25 \sigma^{4} x^{2}(0.5 x(t)-1)\right] \mathrm{d} t \\
& +0.5 \sigma^{2} x(t) \mathrm{d} W(t)\left(x^{0.5}(t)-1\right)
\end{aligned}
$$

Worth mentioning,

$$
\begin{aligned}
& 0.5 x^{0.5}(t) \mu_{\max }\left(1-\frac{x(t)}{x_{\max }}\right)-0.5 x^{-0.5}(t) \mu_{\max }\left(1-\frac{x(t)}{x_{\max }}\right) \\
& +0.25 \sigma^{4} x^{2}(0.5 x(t)-1) \\
& \leq 0.5 x^{0.5}(t) \check{\mu}_{\max }\left(1-\frac{x(t)}{x_{\max }}\right)-0.5 x^{-0.5}(t) \check{\mu}_{\max }\left(1-\frac{x(t)}{x_{\max }}\right) \\
& +0.25 \sup _{t \in R^{+}}\left|\sigma^{4}\right| x^{2}(t)(0.5 x(t)-1) .
\end{aligned}
$$

If $x(t) \leq x_{\max }$ then $\left(1-\frac{x(t)}{x_{\max }}\right) \geq 0$, it follows the boundedness $\sigma$. Therefore, there is nonnegative number $Q_{1}$ which independent of $x$ and $t$.

If $0<x(t)<x_{\max }$, obviously there is a positive number $Q_{2}$ which is inde- 
pendent of $x$ and $t$, and it follow bounded $\sigma$, such that

$$
\begin{aligned}
& 0.5 x^{0.5}(t) \mu_{\max }\left(1-\frac{x(t)}{x_{\max }}\right)-0.5 x^{-0.5}(t) \mu_{\max }\left(1-\frac{x(t)}{x_{\max }}\right) \\
& +0.25 \sigma^{4} x^{2}(0.5 x(t)-1) \\
& \leq 0.5 x^{0.5}(t) \hat{\mu}_{\max }\left(1-\frac{x(t)}{x_{\max }}\right)-0.5 x^{-0.5}(t) \hat{\mu}_{\max }\left(1-\frac{x(t)}{x_{\max }}\right) \\
& +0.25 \sup _{t \in R^{+}}\left|\sigma^{4}\right| x^{2}(0.5 x(t)-1) \leq Q_{2} .
\end{aligned}
$$

On the other hand, we show that there is a nonnegative number $Q$ which independent of $x$ and $t$ such that

$$
\begin{aligned}
& 0.5 x^{0.5}(t) \mu_{\max }\left(1-\frac{x(t)}{x_{\max }}\right)-0.5 x^{-0.5}(t)-\mu_{\max }\left(1-\frac{x(t)}{x_{\max }}\right) \\
& +0.25 \sigma^{4} x^{2}(0.5 x(t)-1) \leq Q .
\end{aligned}
$$

By substituting the inequality (19) into Equation (13) yields

$$
\begin{aligned}
\mathrm{d}(V(x(t))) \leq & Q \mathrm{~d} t+0.5 \sigma x(t) b W(t)\left(1-x^{0.5}(t)(t)\right) \mathrm{d} t \\
& +0.5 \sigma^{2} x(t) \mathrm{d} W(t)\left(x^{0.5}(t)-1\right) .
\end{aligned}
$$

where $W(t)$ is Brownian motion, and taking integral and expectation

$$
E\left(V\left(x\left(i_{n} \wedge T\right)\right)\right) \leq V(x(0))+Q E\left(i_{n} \wedge T\right) \leq V(x(0))+Q T .
$$

By taking into account, the inequality (11) then we have

$$
P\left(i_{n} \leq T\right) \geq \epsilon \text {. }
$$

For every $\epsilon \in\left(i_{n} \leq T\right), x\left(i_{n}, \omega\right)=n$ or $(i, \omega)=\frac{1}{n}$. Therefore, $V(x(t))$ is no less than either

$$
\min \left\{\sqrt{k}-1-\frac{1}{2} \ln (k), \frac{1}{\sqrt{k}}-1+\frac{1}{2} \ln (k)\right\} .
$$

Based on (20) we have

$$
\begin{aligned}
V(x(0))+Q T & \geq E\left[\left\{i_{n} \leq T\right\}(\omega) V\left(x\left(i_{n}\right)\right)\right] \\
& \geq \epsilon \min \left\{\sqrt{k}-1-\frac{1}{2} \ln (k), \frac{1}{\sqrt{k}}-1+\frac{1}{2} \ln (k)\right\} .
\end{aligned}
$$

where $\left\{i_{n} \leq T\right\}(\omega)$ illustrates the function $\left\{i_{n} \leq T\right\}$. Letting $n$ tends to infinity leads to dissidence

$$
\infty>V(x(0))+Q T=\infty
$$

Therefore, we need to have $i_{\infty}=\infty$. Eventually, our claim is proved truly.

In the next section we prove that Equation (10) with Ornstein-Uhlenbeck process is globally asymptotically stable in positive solution which illustrates the highly randomness in stationary phase.

Theorem 3.2: Equation (10) is globally asymptotically stable in positive equi- 
librium points $x_{\max }$, under following assumptions a.s.

H1: For

1) $0 \leq t_{0}, 0<x_{0}<\sigma<\mu_{\text {max }}<b<x_{\text {max }}$

2) $0 \leq t_{0}, 0<x_{0}<\sigma<b<x_{\max }<\mu_{\max }$

and the

$$
\limsup _{t \rightarrow \infty} \frac{\int_{0}^{t} x(s) \mathrm{d} s}{t}=\check{b} \text { and } \liminf _{t \rightarrow \infty} \frac{\int_{0}^{t} x(s) \mathrm{d} s}{t}=\hat{b} .
$$

where, $\breve{b}$ and $\hat{b}$ are positive constant then the solution $x(t)$ satisfies the $\lim _{t \rightarrow \infty} x(t)=x_{\max }$.

Proof: An application Itô's formula ([27], p. 95) [28] [29] [30] for Equation (10) and somewhat lengthy calculation leads to

$$
\begin{aligned}
x(t)= & \frac{x_{\max }}{1+\left[x_{0} \exp \left(\sigma b \int_{0}^{t} x(s) W(s) \mathrm{d} s+\sigma^{2} \int_{0}^{t} x(s) \mathrm{d} W(s)\right)\right.} \\
& \times \frac{1}{\left.\exp \left(-\frac{\mu_{\max }}{x_{\max }} \int_{0}^{t} x(s) \mathrm{d} s-\frac{\sigma^{4}}{2} \int_{0}^{t} x^{2}(s) \mathrm{d} s-\mu_{\max } \int_{0}^{t} \mathrm{~d} s\right)\left(\frac{x_{\text {max }}}{x_{0}}-1\right)\right]}
\end{aligned}
$$

For the convenience we set the denominator of Equation (22) to $Q$ and according to the ([10], p. 69), we have:

$$
\begin{aligned}
Q= & 1+\mathrm{e}^{\left(-\int_{0}^{t} \mu_{\max } \mathrm{d} s-\frac{\mu_{\max }}{x_{\max }} \int_{0}^{t} x(s) \mathrm{d} s-\int_{0}^{t} \sigma b W(s) \mathrm{d} W(s) \times \int_{0}^{t} x(s) \mathrm{d} W(s)\right)} \\
& \times \mathrm{e}^{\left(\sigma^{2} \int_{0}^{t} x(s) \mathrm{d} W(s)-\frac{1}{2} \sigma^{4} \int_{0}^{t} x^{2}(s) \mathrm{d} s\right)} .
\end{aligned}
$$

Taking limit from both sides of above equation yields:

$$
\begin{aligned}
\lim _{t \rightarrow \infty} Q= & 1+\lim _{t \rightarrow \infty} \mathrm{e}^{(-t)\left(\frac{\int_{0}^{t} \mu_{\max } \mathrm{d} s}{t}+\frac{\mu_{\max }}{x_{\max }} \int_{0}^{t} x(s) \mathrm{d} s\right.}+\frac{\int_{0}^{t} \sigma b W(s) \mathrm{d} W(s) \int_{0}^{t} x(s) \mathrm{d} W(s)}{t} \\
& \times \lim _{t \rightarrow \infty} \mathrm{e}^{\left.(-t)-\sigma^{-} \frac{\int_{0}^{t} x(s) \mathrm{d} W(s)}{t}+\frac{1}{2} \sigma^{4} \frac{\int_{0}^{t} x^{2}(s) \mathrm{d} s}{t}\right)} .
\end{aligned}
$$

where,

$$
\int_{0}^{t} x(s) \mathrm{d} W(s)=M(t)
$$

is a Martingale with quadratic variation

$$
\langle M(t), M(t)\rangle=\int_{0}^{t} x^{2}(s) \mathrm{d}(s)
$$

Based on ([31], p. 65) the

$$
\lim _{t \rightarrow \infty} \sup \frac{M(t)}{t}=0 \text { a.s. }
$$

and

$$
\limsup _{t \rightarrow \infty} \frac{\langle M(t), M(t)\rangle}{t}=C \text { a.s. }
$$


where $C \in R^{+}$then Equation (23) becomes

$$
\begin{gathered}
\lim _{t \rightarrow \infty} Q=1+\lim _{t \rightarrow \infty} \mathrm{e}^{(-t)\left(\frac{\int_{0}^{t} \mu_{\max } \mathrm{d} s}{t}+\frac{\mu_{\max }}{x_{\max }} \frac{\int_{0}^{t} x(s) \mathrm{d} s}{t}+\frac{1}{2} \sigma^{4} C\right)} \\
\lim _{t \rightarrow \infty} Q=1+\lim _{t \rightarrow \infty} \mathrm{e}^{(-t)\left(\frac{\int_{0}^{t} \mu_{\max } \mathrm{d} s}{t}+\frac{\mu_{\max }}{x_{\max }} \frac{\int_{0}^{t} x(s) \mathrm{d} s}{t}+\frac{1}{2} \sigma^{4} C\right)}=0 \text { a.s. }
\end{gathered}
$$

By substituting Equation (29) into Equation (22) we have:

$$
\begin{aligned}
& \lim _{t \rightarrow \infty} x(t)=\lim _{t \rightarrow \infty} x_{\max } \\
& \Rightarrow \lim _{t \rightarrow \infty} x(t)=x_{\max } .
\end{aligned}
$$

Ultimately, prove is completed.

Remark 3.1: Liu et al., in [21] studied the stability of stochastic logistic model with white noise which illustrates highly randomness in lag phase and the stationary phase is steady means there is no fluctuations. In lag phase microbes are adapted with each other and as a result no growth takes place. But, the model, conditions and approach which are presented in this study quite different and easy respectively. This research considers Equation (6) with Ornstein-Uhlenbeck process which highly presents the randomness in stationary phase. In stationary phase microbes fight with each other to survive (uncontrolled fluctuations reached maximum level). In addition, concentration of toxin reaches a value which is unable to maintain the maximum cell growth of microorganism rate in fermentation process. These agents become the cause of instability at stationary phase in fermentation process. In conclusion, the stochastic logistic model which presented in Lui et al., [21] is not suitable model for cell growth of microorganism in fermentation process. Since, Lui et al., in [21] narrates in zero solution (almost lag phase in fermentation process) the population (cell growth) is extinctive and in positive equilibrium point (almost stationary phase) the population will be permanent. It is contrary to the features phases in fermentation process. To support our theory, we use the SRK4 method for numerical simulations which presented in below section.

\section{Numerical Simulation}

In this section we consider a strong and accurate numerical method (SRK4) to elaborate the analytical results ([3], p. 69) [4] [5] [6] [7]. Worth mentioning, we cannot use the stochastic Runge-Kutta to approximate the numerical solution of Equation (10), due to it is in Itô sense [33]. Thus, it is convertible to Stratonovich sense by using the below formula

$$
\bar{f}(t, x(t))=f(t, x(t))-\frac{1}{2} g(t, x(t)) \frac{\partial g}{\partial x}(t, x(t))
$$

Hence,

$$
\mathrm{d} x(t)=\left[\mu_{\max }\left(1-\frac{x(t)}{x_{\max }}\right) x(t)-\sigma x^{2}(t) b W(t)\right] \mathrm{d} t-\sigma^{4} x^{3}(t) \circ \mathrm{d} W(t)
$$


where $\circ$ is used to denote the Stratonovich form of SDE (i.e. $\circ \mathrm{d} W(t)$. Equation (10) and Equation (31) present some solution under different approach. We use the SRK4 method for numerical approximation. This method was introduced by Rümellin [8]. SRK was developed based on the increment of Wiener process, $\int_{t_{n}}^{t_{n+1}} \circ \mathrm{d} W(t)$. Furthermore, Xiao in [9] introduced the High strong order stochastic Runge-Kutta methods for stochastic differential equations in case of Stratonovich with scalar noise. Since our model is in Stratonovich sense. Figure 1 and Figure 2 show the stability of Equation (3) and Equation (10) in positive equilibrium for cell growth of microorganism in fermentation process respectively.

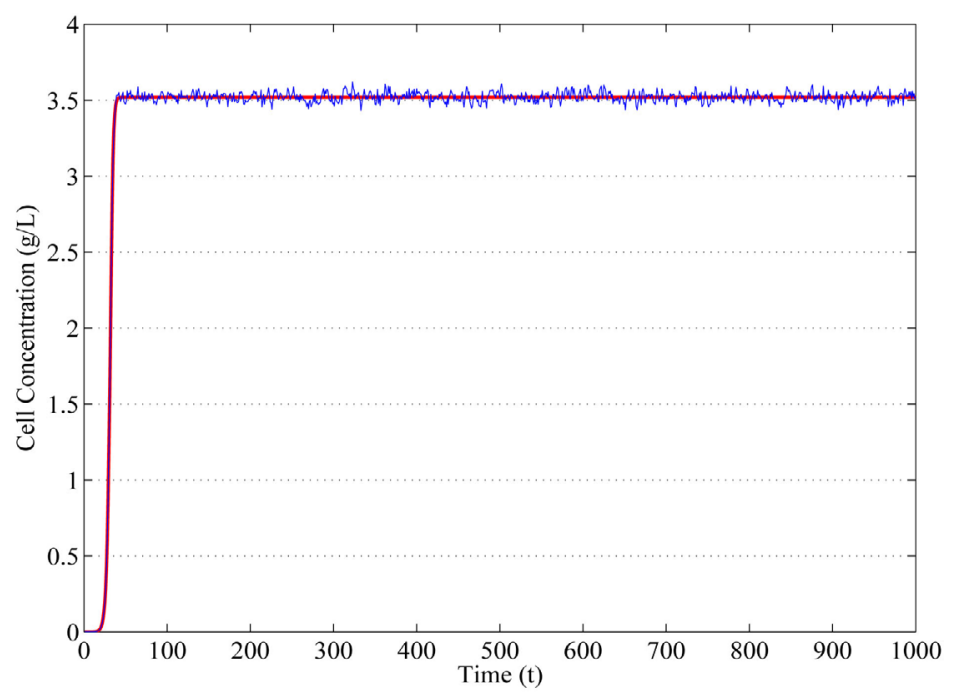

Figure 1. Shows the stability of Equation (10). For deterministic part we use $t_{0}=0.00$, $x(0)=0.0001, x_{\max }=3.520, \mu_{\max }=0.5, \sigma=0.00$, and $b=0$ and for stochastic part we chose same value just difference are $\sigma=0.002$, and $b=1$ respectively.

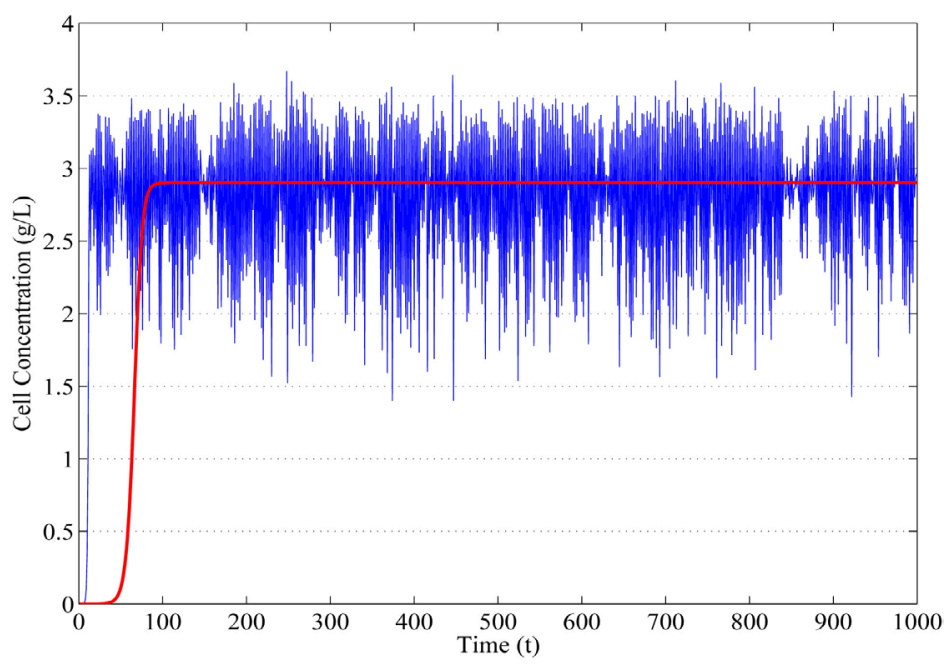

Figure 2. Illustrates the stability of Equation (10). For stochastic part we chose the $t_{0}=0.00, x(0)=0.0001, x_{\max }=3.520, \mu_{\max }=1.99, b=1$, and $\sigma=0.021$. For deterministic part we use $\sigma=0.00, b=0, t_{0}=0.00, x(0)=0.0001, x_{\max }=3.520$, $\mu_{\max }=0.21$. 
The blue line indicates the simple path of Equation (10) and the red line shows the simple path of Equation (3) respectively. In Figure 1 and Figure 2, the difference are between the value of $\sigma, \mu_{\max }$. The blue line indicates the simple path of Equation (10) and the red line shows the simple path of Equation (3) respectively. Figure 1 and Figure 2 are plotted under $\mathrm{H} 1$.

In both Figure 1 and Figure 2 lag phase is the luck of random fluctuations due to microbes are adopt with each other. Therefore, no growth takes place in this phase. Contrariwise, in stationary phase microbes fight with each other for food and space and the behavior of cell proliferation of microorganism is different in phases at fermentation process. As time evolves, the system illustrates the intrinsic variability of the competing within species and deviations from exponential growth arise. It happens as a result of the nutrient level and toxin concentration achieves a value which can no longer support the maximum growth rate. So, the stochastic fluctuations mainly affect the logistic growth of microorganism and for more see Remarks 3.1.

Remark 4.1: The red line shows the simple path of Equation (3) and the blue line indicates the simple path of Equation (10) respectively. If $\sigma \rightarrow \pm \infty$, there is no phases for cell growth which means this random fluctuations will destroy the phases in fermentation process on the other hands the process will tend to infinity.

Remark 4.2: Figures 1-4 show the stability of Equation (3) and Equation (10) in different equilibrium points. Figure 3 and Figure 4 are plotted under H1 option 2. The thick red line illustrates the stability of Equation (10) without Ornstein-Uhlenbeck process. It is thick in stationary phase due to the growth rate $\mu_{\max }$, is increased, compare the value of $\mu_{\max }$ with three others Figures.

\section{Conclusion}

This research is conducted on stability of stochastic logistic model with Ornstein-Uhlenbeck process for cell growth of microorganism in fermentation.

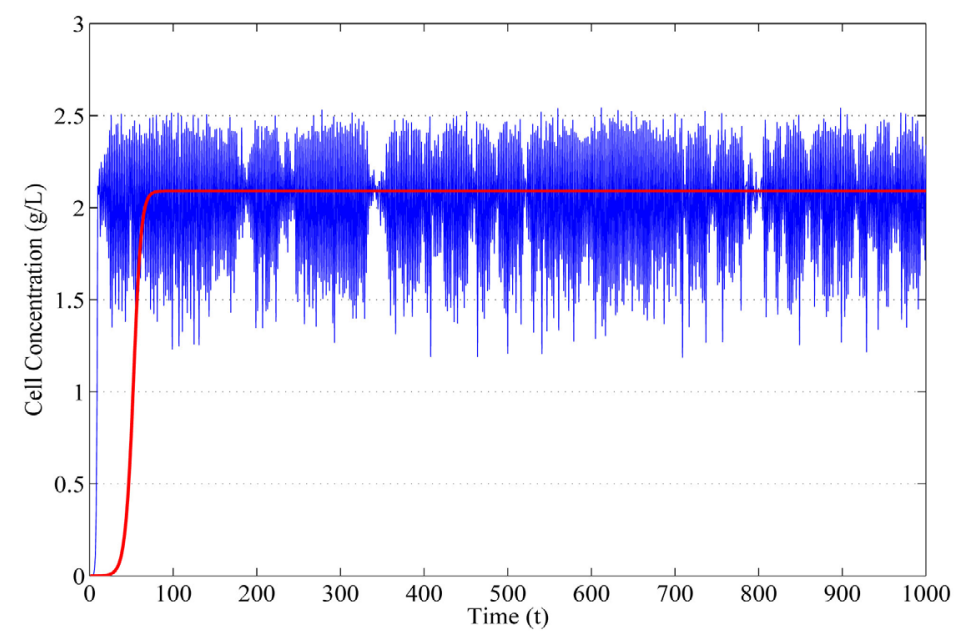

Figure 3. Shows the stability of Equation (10). For deterministic part we use $t_{0}=0.00$, $x(0)=0.0001, x_{\max }=2.09, \mu_{\max }=0.2, \sigma=0.00$, and $b=0$ and for stochastic part we chose same value just difference are $\sigma=0.0021, \mu_{\max }=2.2$ and $b=1$ respectively. 


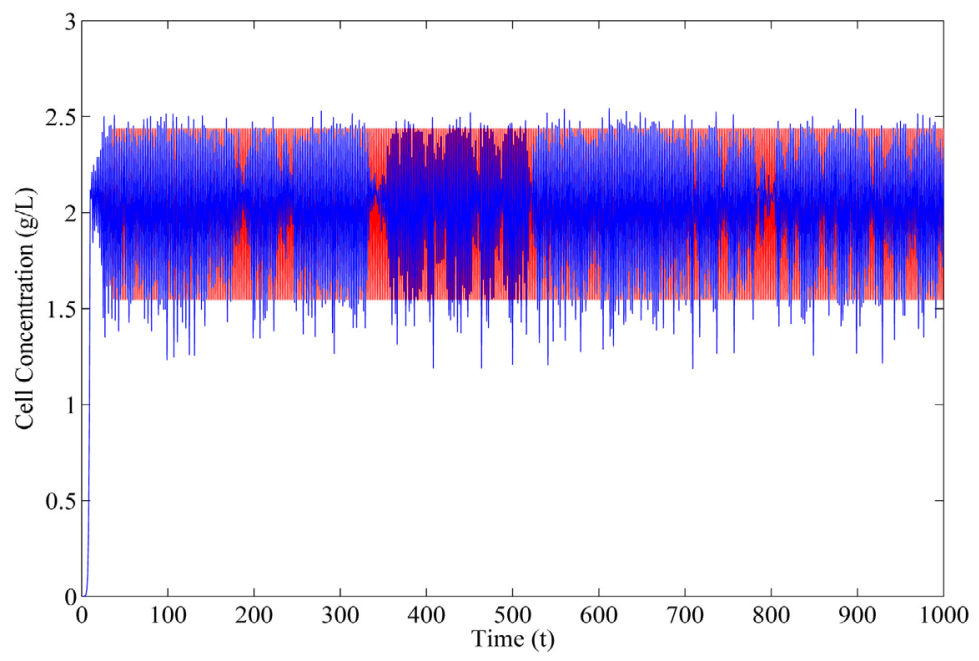

Figure 4. Illustrates the stability of Equation (10). For stochastic part we chose the $t_{0}=0.00, x(0)=0.0001, x_{\max }=2.09, \mu_{\max }=2.2, \mu_{\max }=0.21, b=1$, and $\sigma=0.02$. For deterministic part we use $\sigma=0.00, b=0, t_{0}=0.00, x(0)=0.0001, x_{\max }=2.09$, $\mu_{\max }=2.2$.

This research proved that Equation (10) has a unique positive solution (see Theorem 3.1). Moreover, we proved that Equation (10) was stochastically stable in zero solution and positive equilibrium point (see Theorem 3.2 and Appendix A Theorem 1) and viewed in Figure 1 and Figure 2 and Appendix A. For numerical simulation we used SRK4 method to show the reality of this research. If the $\sigma \rightarrow \pm \infty$, the result will be infinity which means that there are no phases for cell growth. On the other hand, it will not preserve the stochastically stable. If $x_{0}>x_{\max }>\mu_{\max }$, what will happen? Unfortunately, there are some impediments here which need some further investigation in future perspective.

\section{Acknowledgements}

This work is jointly supported by the National Natural Science Foundation of China under Grant Nos. 61573291, the Fundamental Research Funds for Central Universities XDJK2016B036.

\section{Conflicts of Interest}

The author declares no conflicts of interest regarding the publication of this paper.

\section{References}

[1] Ayoubi, T., Rosli, N., Bahar, A. and Salleh, M.M. (2015) Time Delay and Noise Explaining the Behaviour of the Cell Growth in Fermentation Process. AIP Conference Proceedings, 1643, 547-554. https://doi.org/10.1063/1.4907493

[2] Bahar, A. and Mao, X. (2004) Stochastic Delay Population Dynamics. International Journal of Pure and Applied Mathematics, 11, 377-400.

[3] Ayoubi, T. (2015) Stochastic Modelling of Time Delay for Solvent Production by 
Clostridium acetobutylicum. Master Thesis, Universiti Malaysia Pahang, Pahang.

[4] Burrage, K. and Burrage, P.M. (1996) High Strong Order Explicit Runge-Kutta Methods for Stochastic Ordinary Differential Equations. Applied Numerical Mathematics, 22, 81-101. https://doi.org/10.1016/S0168-9274(96)00027-X

[5] Burrage, P.M. (1999) Runge-Kutta Methods for Stochastic Differential Equations. Ph.D. Thesis, University of Queensland, Queensland, Australia.

[6] Ariffin, N.N.A., Rosli, N. and Kasim, A.M. (2017) Stability Analysis of 4-Stage Stochastic Runge-Kutta Method (SRK4) and Specific Stochastic Runge-Kutta Method (SRKS1.5) for Stochastic Differential Equations. In: Kor, L.K., Ahmad, A.R., Idrus, Z. and Mansor, K., Eds., Proceedings of the 3rd International Conference on Computing, Mathematics and Statistics, Springer, Singapore, 187-194. https://doi.org/10.1007/978-981-13-7279-7_23

[7] Napoli, A. (2012) On a Class of Stochastic Runge Kutta Methods. International Journal Contemputation and Mathematics Sciences, 36, 1757-1769.

[8] Rüemelin, W. (1982) Numerical Treatment of Stochastic Differential Equations. SIAM Journal on Numerical Analysis, 19, 604-613. https://doi.org/10.1137/0719041

[9] Xiao, A. and Tang, X. (2016) High Strong Order Stochastic Runge-Kutta Methods for Stratonovich Stochastic Differential Equations with Scalar Noise. Numerical Algorithms, 72, 259-296. https://doi.org/10.1007/s11075-015-0044-0

[10] Evans, L.C. (2012) An Introduction to Stochastic Differential Equations. American Mathematical Society, Providence, RI. https://doi.org/10.1090/mbk/082

[11] Mazo, R.M. (2002) Brownian Motion: Fluctuations, Dynamics, and Applications. Oxford University Press, Oxford.

[12] Mishura, I.S. and Mishura, Y. (2008) Stochastic Calculus for Fractional Brownian Motion and Related Processes. Springer Science and Business Media, Berlin,' Heidelberg. https://doi.org/10.1007/978-3-540-75873-0

[13] Madihah, M.S., Ariff, A.B., Khalil, M.S., Suraini, A.A. and Karim, M.I.A. (2001) Anaerobic Fermentation of Gelatinized Sago Starch-Derived Sugars to Acetone-1-Butanol-Ethanol Solvent by Clostridium acetobutylicum. Folia Microbiologica, 46, 197-204. https://doi.org/10.1007/BF02818533

[14] Madihah, M.S. (2002) Direct Fermentation of Gelatinised Sago Starch to Solvent (Actone, Butanol and Ethanol) by Clostridium acetobutylicum. Ph.D. Thesis, Universiti Putra Malaysia, [Serdang, Selangor, Malaysia.

[15] Bazli, M.K. (2010) Stochastic Modelling of the Clostridium acetobutylicum and Solvent Productions in Fermentation. Master Thesis, Universiti Technology Malaysia, Malaysia.

[16] Aruğaslan, D. and Güzel, L. (2015) Stability of the Logistic Population Model with Generalized Piecewise Constant Delays. Advances in Difference Equations, 1, 173. https://doi.org/10.1186/s13662-015-0521-8

[17] Jiang, D., Shi, N. and Li, X. (2008) Global Stability and Stochastic Permanence of a Non-Autonomous Logistic Equation with Random Perturbation. Journal of Mathematical Analysis and Applications, 340, 588-597. https://doi.org/10.1016/j.jmaa.2007.08.014

[18] Liu, M. and Bai, C. (2015) A Remark on a Stochastic Logistic Model with Lévy Jumps. Applied Mathematics and Computation, 251, 521-526. https://doi.org/10.1016/j.amc.2014.11.094

[19] Liu, Q. and Chen, Q. (2016) Analysis of a General Stochastic Non-Autonomous Logistic Model with Delays and Lévy Jumps. Journal of Mathematical Analysis and 
Applications, 433, 95-120. https://doi.org/10.1016/j.jmaa.2015.07.030

[20] Liu, Q., Jiang, D., Hayat, T. and Alsaedi, A. (2018) Long-Time Behavior of a Stochastic Logistic Equation with Distributed Delay and Nonlinear Perturbation. Physic A: Statistical Mechanics and Its Applications, 508, 289-304. https://doi.org/10.1016/j.physa.2018.05.054

[21] Liu, M. and Wang, K. (2013) A Note on Stability of Stochastic Logistic Equation. Applied Mathematics Letters, 26, 601-606. https://doi.org/10.1016/j.aml.2012.12.015

[22] Mateos-Núñez, D. and Cortés, J. (2013) Stability of Stochastic Differential Equations with Additive Persistent Noise. 2013 American Control Conference, Washington DC, 17-19 June 2013, 5427-5432. https://doi.org/10.1109/ACC.2013.6580686

[23] Murray, J.D. (2001) Mathematical Biology II. Spatial Models and Biomedical Applications. In: Interdisciplinary Applied Mathematics, Springer-Verlag, New York, $1-6$

[24] May, R.M. (2001) Stability and Complexity in Model Ecosystems. Princeton University Press, Princeton, NJ.

[25] Arnold, L. (1972) Stochastic Differential Equations: Theory and Applications. Wiley, Hoboken, NJ.

[26] Friedman, A. (1976) Stochastic Differential Equations and Their Applications. Academic Press, Cambridge, MA.

[27] Allen, E. (2007) Modeling with Itô Stochastic Differential Equations. Springer Science and Business Media, Berlin, Heidelberg.

[28] Khasminskii, R. (2011) Stochastic Stability of Differential Equations. Springer Science and Business Media, Berlin,', Heidelberg.

[29] Mao, X. (1991) Stability of Stochastic Differential Equations with Respect to Semi-Martingales. Longman, Harlow.

[30] Mao, X. (1994) Exponential Stability of Stochastic Differential Equations. Marcel Dekker, New York.

[31] Mao, X. (2007) Stochastic Differential Equations and Applications. Elsevier, Amsterdam, Netherlands.

[32] Gardiner, C.W. (2004) Handbook of Stochastic Methods for Physics, Chemistry and the Natural Sciences (Springer Series in Synergetics). Springer, Berlin’, Heidelberg.

[33] Rosli, N., Bahar, A., Su Hoe, Y. and Abd Rahman, H. (2010) Stochastic Model of Gelatinised Sago Starch to Solvent Production by C. Acetobutylicum P262. In: Proceedings of the Regional Conference on Statistical Sciences 2010, Shah Alam, Selangor, 9-20. 


\section{Appendix A}

Theorem 1: Equation (10) under the following assumption is globally asymptotically stable in zero solution a.s (almost surely).

(H2): For $0 \leq t_{0}, \mu_{\max }<0<x_{0}<\sigma<x_{\max }$, then the $\lim _{t \rightarrow \infty} x(t)=0$.

Proof: An application of Itô's formula for Equation (10) ([27], p. 95) leads to

$$
\begin{aligned}
& \mathrm{d}(\ln |x(t)|) \\
& =\frac{1}{x(t)} \underbrace{\left[\mu_{\max }\left(1-\frac{x(t)}{x_{\max }}\right) x(t) \mathrm{d} t-\sigma x^{2}(t) b W(t) \mathrm{d} t+\sigma^{2} x^{2}(t) \mathrm{d} W(t)\right]}_{A} \\
& +\frac{-1}{2 x^{2}(t)} \underbrace{\left[\mu_{\max }\left(1-\frac{x(t)}{x_{\max }}\right) x(t) \mathrm{d} t-\sigma x^{2}(t) b W(t) d t+\sigma^{2} x^{2}(t) \mathrm{d} W(t)\right]^{2}}_{B}
\end{aligned}
$$

Equation (32) has two parts $A$ and $B$.

So,

$$
A=\mu_{\max }\left(1-\frac{x(t)}{x_{\max }}\right) x(t) \mathrm{d} t-\sigma x^{2}(t) b W(t) \mathrm{d} t+\sigma^{2} x^{2}(t) \mathrm{d} W(t)
$$

and

$$
B=\left[\mu_{\max }\left(1-\frac{x(t)}{x_{\max }}\right) x(t) \mathrm{d} t-\sigma x^{2}(t) b W(t) \mathrm{d} t+\sigma^{2} x^{2}(t) \mathrm{d} W(t)\right]^{2} .
$$

To simplify the $B$ we have

$$
\begin{gathered}
B=\left[\mu_{\max }\left(1-\frac{x(t)}{x_{\text {max }}}\right) \mathrm{d} t-\sigma x(t) b W(t) \mathrm{d} t+\sigma^{2} x^{2}(t) \mathrm{d} W(t)\right]^{2} \\
B=\left(\mu_{\text {max }}\left(1-\frac{x(t)}{x_{\text {max }}}\right) \mathrm{d} t\right)^{2}+2\left(\mu_{\text {max }}\left(1-\frac{x(t)}{x_{\text {max }}}\right) \mathrm{d} t\right) \\
\times\left(-\sigma x(t) b W(t) \mathrm{d} t+\sigma^{2} x^{2}(t) \mathrm{d} W(t)\right) \\
+\left(-\sigma x(t) b W(t) \mathrm{d} t+\sigma^{2} x^{2}(t) \mathrm{d} W(t)\right)^{2} \\
B=\left(\mu_{\text {max }}^{2} \mathrm{~d} t \times \mathrm{d} t-2 \frac{\mu_{\text {max }}}{x_{\text {max }}} x^{2}(t) \mathrm{d} t \times \mathrm{d} t+\left(\frac{x(t)}{x_{\max }} \mathrm{d} t\right)^{2}\right) \\
+2\left(\mu_{\text {max }} \mathrm{d} t-\frac{\mu_{\text {max }}}{x_{\text {max }}} x^{2}(t) \mathrm{d} t\right) \times\left(-\sigma x(t) b W(t) \mathrm{d} t+\sigma^{2} x(t) \mathrm{d} W(t)\right) \\
+\left(-\sigma x(t) b W(t) \mathrm{d} t+\sigma^{2} x(t) \mathrm{d} W(t)\right)^{2}+(-\sigma x(t) b W(t) \mathrm{d} t)^{2} \\
+2(-\sigma x(t) b W(t) \mathrm{d} t)\left(\sigma^{2} x(t) \mathrm{d} W(t)\right)+\left(\sigma^{2} x^{2}(t) \mathrm{d} W(t)\right)^{2}
\end{gathered}
$$

Using these facts $\mathrm{d} t \times d t=0, \mathrm{~d} t \times \mathrm{d} w(t)=0$ and $\mathrm{d} W(t) \times \mathrm{d} W(t)=\mathrm{d} t \quad$ ([32], . 87) and somewhat lengthy calculation we have

$$
B=\sigma^{4} x^{4}(t) \mathrm{d} t .
$$

By substituting the value of A and B into Equation (32) yields: 


$$
\begin{array}{r}
\mathrm{d}(\ln |x(t)|)=\frac{1}{x(t)}\left[\mu_{\max }\left(1-\frac{x(t)}{x_{\max }}\right) x(t) \mathrm{d} t-\sigma x^{2}(t) b W(t) \mathrm{d} t\right. \\
\left.+\sigma^{2} x^{2}(t) \mathrm{d} W(t)\right]+\frac{-1}{2 x(t)^{2}}\left(\sigma^{4} x^{4}(t) \mathrm{d} t\right) \\
\mathrm{d}(\ln |x(t)|)=\left[\mu_{\max }\left(1-\frac{x(t)}{x_{\max }}\right) \mathrm{d} t-\sigma x(t) b W(t) \mathrm{d} t\right. \\
\left.+\sigma^{2} x(t) \mathrm{d} W(t)\right]-\frac{1}{2}\left(\sigma^{4} x^{2}(t) \mathrm{d} t\right) \\
x(t)=\mathrm{e}^{\mu_{\max } \int_{0}^{t}\left(1-\frac{x(s)}{x_{\max }}\right) \mathrm{d} s-\sigma b \int_{0}^{t} x(s) W(s) \mathrm{d}+\sigma^{2} \int_{0}^{t} x(s) \mathrm{d} W(s)-\frac{1}{2} \sigma^{4} \int_{0}^{t} x^{2}(s) \mathrm{d} s}
\end{array}
$$

where $W(t)$ is Brownian motion and taking expectation from both sides of above equation. We know the expectation of Brownian motion is zero ([10], Section 3.2.3, p. 43), we get

$$
E[x(t)]=E\left[\exp \left(-\frac{\mu_{\max }}{x_{\max }} \int_{0}^{t} x(s) \mathrm{d} s-\frac{\sigma^{4}}{2} \int_{0}^{t} x^{2}(s) \mathrm{d} s+\mu_{\max } \int_{0}^{t} \mathrm{~d} s\right)\right]
$$

Takes limit

$$
\begin{aligned}
& \lim _{t \rightarrow \infty} E[x(t)]=\lim _{t \rightarrow \infty} E\left[\exp \left(-\frac{\mu_{\max }}{x_{\max }} \int_{0}^{t} x(s) \mathrm{d} s-\frac{\sigma^{4}}{2} \int_{0}^{t} x^{2}(s) \mathrm{d} s+\mu_{\max } \int_{0}^{t} \mathrm{~d} s\right)\right] \\
& \lim _{t \rightarrow \infty} E[x(t)]=\lim _{t \rightarrow \infty} E\left[\exp \left(-\frac{\mu_{\max }}{x_{\max }} \frac{\int_{0}^{t} x(s) \mathrm{d} s}{t}-\frac{\sigma^{4}}{2} \frac{\int_{0}^{t} x^{2}(s) \mathrm{d} s}{t}+\mu_{\max } \frac{\int_{0}^{t} \mathrm{~d} s}{t}\right)\right]
\end{aligned}
$$

For $0 \leq t \leq T$ and based on ([10], p. 66 and p. 69) the $\int_{0}^{T} x^{2}(s) \mathrm{d} s<\infty$ and $\int_{0}^{T} x(s) \mathrm{d} s<\infty$. Therefore

$$
\lim _{t \rightarrow \infty} E\left[\mathrm{e}^{\left(\mu_{\max }^{\int_{0}^{t} \mathrm{ds}} \frac{t}{t}\right)}\right]
$$

Under $\mathrm{H} 2$ yields

$$
\lim _{t \rightarrow \infty} E\left[\mathrm{e}^{t\left(\mu_{\max } \frac{\int_{0}^{t} \mathrm{~d} s}{t}\right)}\right]=0 .
$$

Therefore, Equation (38) becomes

$$
\lim _{t \rightarrow \infty} x(t)=0 .
$$

Ultimately, our claim is proved.

For plotting Figure 5 we use $t_{0}=0.00, x(0)=0.0001, x_{\max }=3.5250$, $\mu_{\max }=-0.0091, \sigma=0.00, \sigma=3.5$ and $b=0, b=90$ and for Figure 6 we use $t_{0}=0.00, x(0)=0.0001, x_{\max }=0.50, \mu_{\max }=-1.99, \sigma=0.00, \sigma=11$ and $b=0, b=100$. The blue line indicates the simple path of Equation (10) and the red line shows the simple path of Equation (3). 


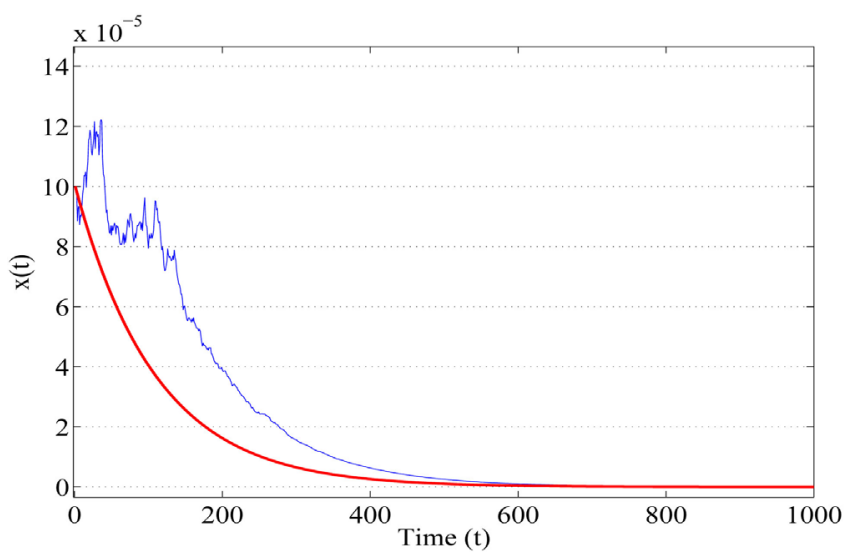

Figure 5. Shows the stability of Equation (10) with different value of parameters.

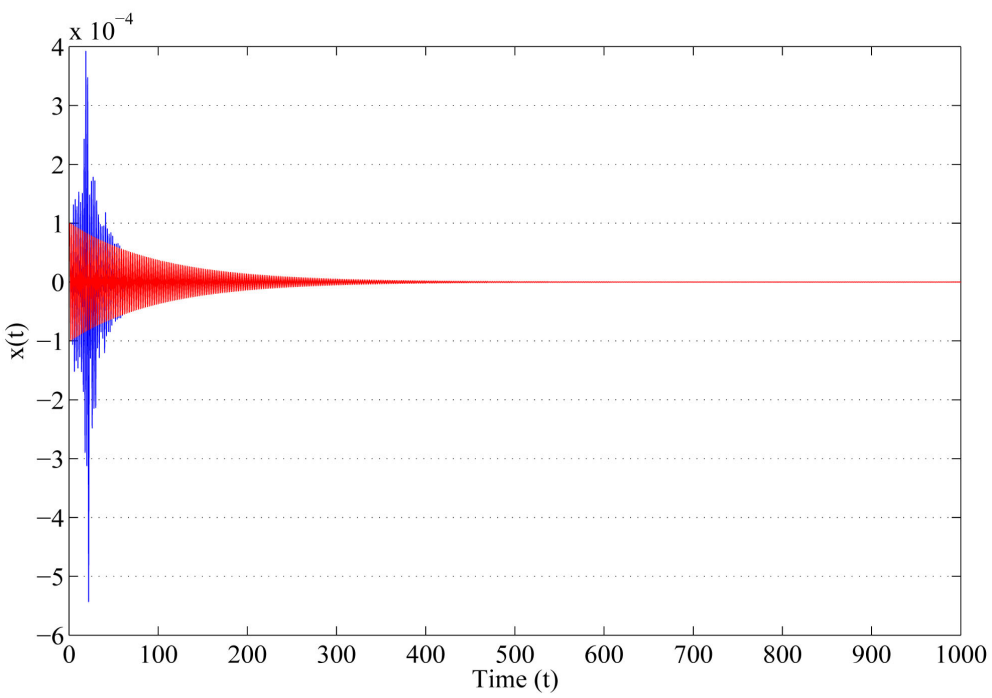

Figure 6. Illustrates the stability of Equation (10) with different value of parameters.

Note: The thick red line shows the stability of Equation (10) without Ornstein-Uhlenbeck process. It is thick due to the growth rate $\mu_{\max }$, is increased, compare the value of $\mu_{\max }$ with Figure 5 . 